\title{
MINIMUM CROSSINGS IN JOIN OF GRAPHS WITH PATHS AND CYCLES
}

\author{
Marián KLEŠČ, Matúš VALO \\ Department of Mathematics and Theoretical Informatics, Faculty of Electrical Engineering and Informatics, Technical University \\ of Košice, Letná 9, 04200 Košice, Slovak Republic, e-mail: marian.klesc@ @uke.sk, matus.valo@ tuke.sk
}

\begin{abstract}
The crossing number $\operatorname{cr}(G)$ of a graph $G$ is the minimal number of crossings over all drawings of $G$ in the plane. Only few results concerning crossing numbers of graphs obtained as join product of two graphs are known. There was collected the exact values of crossing numbers for join of all graphs of at most four vertices and of several graphs of order five with paths and cycles. We extend these results by giving the crossing numbers for join products of the special graph on six vertices with $n$ isolated vertices as well as with the path on $n$ vertices and with the cycle on $n$ vertices.
\end{abstract}

Keywords: graph, drawing, join product, crossing number, path, cycle

\section{INTRODUCTION}

Let $G$ be a graph with vertex set $V(G)$ and edge set $E(G)$. A drawing of $G$ is a representation of $G$ in the plane such that its vertices are represented by distinct points and its edges by simple continuous arcs connecting the corresponding point pairs. For simplicity, we assume that in a drawing (a) no edge passes through any vertex other than its end-points, (b) no two edges touch each other (i.e., if two edges have a common interior point, then at this point they properly cross each other), and (c) no three edges cross at the same point. The crossing number $\operatorname{cr}(G)$ is the smallest number of edge crossings in any drawing of $G$. It is easy to see that a drawing with minimum number of crossings (an optimal drawing) is always a good drawing, meaning that no edge crosses itself, no two edges cross more than once, and no two edges incident with the same vertex cross.

Finding the crossing number of a graph is useful in many areas. The most prominent area is VLSI technology. The lower bound on the chip area is determined by crossing number and by number of vertices of the graph [2]. It plays an important role in various fields of discrete/computational geometry [19]. Crossing number is also parameter yielding the deviation of the graph from being planar. The crossing number influences significantly readability and therefore it is the most important parameter when aesthetics of the graph is considered. It is used mostly in automated visualisation of graphs.

The investigation on the crossing numbers of graphs is a classical and very difficult problem. It was proved by Garey and Johnson [5] that finding the crossing number of a general graph is NP-hard and it remains NP-hard even for cubic graphs $[8]$. Pioneering papers about crossing numbers were published in the second half of the twentieth century. Turán formulated The brick factory problem, which arose during World War II., by publishing in the article [20]. Given problem was a graph problem where kilns and storage yards are vertices and the rails between kilns and storage yards are edges in the graph. Turán asked whether the number of crossings of the rails could be minimized. He realized after several days that the actual situation could be improved, but the exact solution of the general problem with $m$ kilns and $n$ storage yards seemed to be very difficult. Using the construction of suitable diagram of the complete bipartite graph $K_{m, n}$, Zarankiewicz [22] gave the upper bound of the crossing number of the graph $K_{m, n}$ and conjectured that

$\operatorname{cr}\left(K_{m, n}\right)=\left\lfloor\frac{m}{2}\right\rfloor\left\lfloor\frac{m-1}{2}\right\rfloor\left\lfloor\frac{n}{2}\right\rfloor\left\lfloor\frac{n-1}{2}\right\rfloor$.

The given problem has not been generally solved. Kleitman [10] confirmed Zarankiewicz's conjecture for every $n$ and $1 \leq m \leq 6$. Woodall proved that for $7 \leq m \leq 8$ and $7 \leq n \leq 10$, the crossing number of $K_{m, n}$ is equal to Zarankiewicz number, too [21].

The exact values of crossing numbers are known only for several specific families of graphs. The Cartesian product (the definition see in [1]) of two graphs is one of them. Let $C_{n}$ be the cycle of length $n, P_{n}$ be the path of $n$ vertices, and $S_{n}$ be the star isomorphic to $K_{1, n}$. Harary et al. [7] conjectured that the crossing number of the Cartesian product $C_{m} \square C_{n}$ of two cycles is $(m-2) n$, for all $m, n$ satisfying $3 \leq m \leq n$. It was proved by Glebsky and Salazar [6] that for any fixed $m$, the conjecture holds for all $n \geq m(m+1)$. The conjecture has also been verified for $m \leq 7$. Besides of Cartesian product of two cycles, there are several other exact results. In [1,9], the crossing numbers of $G \square C_{n}$ for all graphs $G$ of order at most four are established. In addition, the crossing numbers of $G \square C_{n}$ are known for some graphs $G$ on five or six vertices [4, 16]. Bokal in [3] confirmed the general conjecture for crossing numbers of Cartesian products of paths and stars formulated in [9]. Crossing numbers of Cartesian products of stars and paths with graphs of order at most five were studied in [9.11 12]. The table in [13] shows the summary of known crossing numbers for Cartesian products of path, cycle and star with connected graphs of order five.

The Join product $G_{i}+G_{j}$ of graphs $G_{i}$ and $G_{j}$ is created from vertex-disjoint copies of $G_{i}$ and $G_{j}$ by adding all edges between $V\left(G_{i}\right)$ and $V\left(G_{j}\right)$. For $\left|V\left(G_{i}\right)\right|=m$ and $\left|V\left(G_{j}\right)\right|=n$, the edge set of the graph $G_{i}+G_{j}$ is the union of $E\left(G_{i}\right), E\left(G_{j}\right)$ and $E\left(K_{m, n}\right)$. Let us denote by $D_{n}$ the discrete graph on $n$ vertices. The crossing numbers for join of two paths, join of two cycles, and for join of path and cycle were studied in [14]. Moreover, the exact values for crossing numbers of $G+D_{n}$ and $G+P_{n}$ for all graphs $G$ of order at most four are given in [17]. The crossing num- 
bers of the graphs $G+P_{n}$ and $G+C_{n}$ are also known for very few graphs $G$ of order five and six, see [15, 18].

Let $D(D(G))$ be a good drawing of the graph $G$. The number edge crossings of the graph $G$ in $D$ is denoted by $\operatorname{cr}_{D}(G)$. For edge-disjoint subgraphs $H_{i}$ and $H_{j}$ of $G$, we denote by $\operatorname{cr}_{D}\left(H_{i}, H_{j}\right)$ the number of crossings between the edges of $H_{i}$ and the edges of $H_{j}$ in $D$ and by $\operatorname{cr}_{D}\left(H_{i}\right)$ the number of crossings among edges of $H_{i}$ in $D$. For any three edge-disjoint subgraphs $H_{i}, H_{j}$ and $H_{k}$ of the graph $G$ the following equations hold:

$$
\begin{aligned}
& \operatorname{cr}_{D}\left(H_{i} \cup H_{j}\right)=\operatorname{cr}_{D}\left(H_{i}\right)+\operatorname{cr}_{D}\left(H_{j}\right)+\operatorname{cr}_{D}\left(H_{i}, H_{j}\right), \\
& \operatorname{cr}_{D}\left(H_{i} \cup H_{j}, H_{k}\right)=\operatorname{cr}_{D}\left(H_{i}, H_{k}\right)+\operatorname{cr}_{D}\left(H_{j}, H_{k}\right) .
\end{aligned}
$$

In the proofs of the paper, we will often use the term "region" also in nonplanar drawings. In this case, crossings are considered to be vertices of the "map".

Let $G$ be a graph consisting of one cycle abcdea, one additional vertex $f$ and two edges $a f$ and $d f$. For brevity, let $C_{5}^{e}(G), C_{5}^{f}(G)$, and $C_{4}(G)$ denote the cycles abcdea, $a b c d f a$ and $a f d e a$, respectively. The aim of this paper is to give the crossing numbers of join products of the graph $G$ with the discrete graph $D_{n}$ as well as with the path $P_{n}$ and the cycle $C_{n}$.

\section{THE CROSSING NUMBER OF $G+D_{N}$}

The graph $G+D_{n}$ consists of one copy of the graph $G$ and $n$ vertices $t_{1}, t_{2}, \ldots, t_{n}$, where every vertex $t_{i}, i=$ $1,2, \ldots, n$, is adjacent to six vertices of $G$, see Fig. 1 . For $i=1,2, \ldots, n$, let $T^{i}$ denote the subgraph induced by six edges incident with the vertex $t_{i}$ and let $F^{i}=G \cup T^{i}$. To simplify the notation, let $G(n)$ denote the graph $G+D_{n}$, in this paper. In Fig. 1 one can easily see that

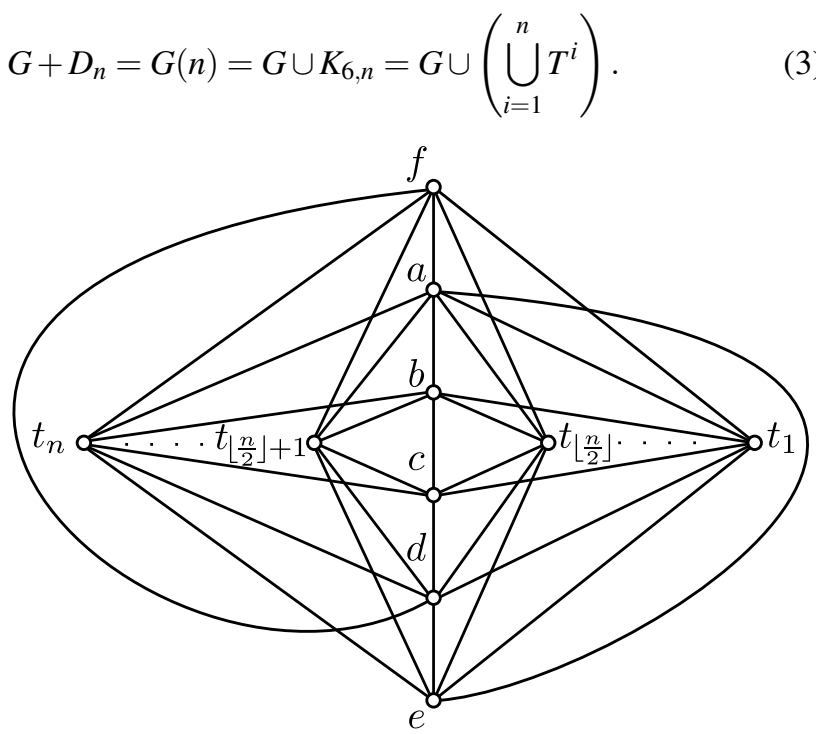

Fig. 1 A drawing of $G+D_{n}$ with $6\left\lfloor\frac{n}{2}\right\rfloor\left\lfloor\frac{n-1}{2}\right\rfloor+n$ crossings.

Theorem 2.1. $\operatorname{cr}\left(G+D_{n}\right)=6\left\lfloor\frac{n}{2}\right\rfloor\left\lfloor\frac{n-1}{2}\right\rfloor+n$ for $n \geq 1$.

Proof. The drawing in Fig. 1 shows that the inequality $\operatorname{cr}\left(G+D_{n}\right) \leq 6\left\lfloor\frac{n}{2}\right\rfloor\left\lfloor\frac{n-1}{2}\right\rfloor+n$ holds. We prove the reverse inequality by induction on $n$.
The graph $G+D_{1}$ contains a subdivision of the complete bipartite graph $K_{3,3}$, and therefore $\operatorname{cr}\left(G+D_{1}\right)=1$. For $n=2, \operatorname{cr}\left(G+D_{2}\right) \geq 2$ since removing any edge of the graph $G+D_{2}$ results in a graph containing a subdivision of $K_{3,3}$. Hence, the assertion of the Theorem is true for $n=1$ and $n=2$.

Suppose now that for $n \geq 3$

$\operatorname{cr}(G(n-2)) \geq 6\left\lfloor\frac{n-2}{2}\right\rfloor\left\lfloor\frac{n-3}{2}\right\rfloor+n-2$

and consider such a drawing $D$ of the graph $G(n)$ that

$\operatorname{cr}_{D}(G(n))<6\left\lfloor\frac{n}{2}\right\rfloor\left\lfloor\frac{n-1}{2}\right\rfloor+n$.

Assume first that there are two different subgraphs $T^{i}$ and $T^{j}$ that do not cross each other in $D$. Without loss of generality, let $\operatorname{cr}_{D}\left(T^{1}, T^{2}\right)=0$. The subdrawing of $D$ induced by $T^{1} \cup T^{2}$ divides the plane in such a way that there are two vertices of $G$ on the boundary of every region. The graph $G$ contains two edge disjoint stars $S_{3}^{a}$ and $S_{3}^{d}$ induced by the vertices $a, b, e, f$ and $d, c, e, f$, respectively. As each region of $D\left(T^{1} \cup T^{2}\right)$ contains exactly two vertices of $G$ on its boundary, $\operatorname{cr}_{D}\left(S_{3}^{x}, T^{1} \cup T^{2}\right) \geq 1, x=a, d$, and therefore $\operatorname{cr}_{D}\left(G, T^{1} \cup T^{2}\right) \geq 2$. Moreover, as $\operatorname{cr}\left(K_{6,3}\right)=6$, in $D$, every subgraph $T^{k}, k=3,4, \ldots, n$, crosses $T^{1} \cup T^{2}$ at least six times. Since $G(n)=G+D_{n}=G(n-2) \cup\left(T^{1} \cup T^{2}\right)$ and $G(n-2)=K_{6, n-2} \cup G$, using (2) and (4) we have

$$
\begin{aligned}
\operatorname{cr}_{D}(G(n)) & =\operatorname{cr}_{D}(G(n-2))+\operatorname{cr}_{D}\left(T^{1} \cup T^{2}\right)+ \\
& +\operatorname{cr}_{D}\left(K_{6, n-2}, T^{1} \cup T^{2}\right)+\operatorname{cr}_{D}\left(G, T^{1} \cup T^{2}\right) \\
& \geq 6\left\lfloor\frac{n-2}{2}\right\rfloor\left\lfloor\frac{n-3}{2}\right\rfloor+(n-2)+ \\
& +0+6(n-2)+2 \geq 6\left\lfloor\frac{n}{2}\right\rfloor\left\lfloor\frac{n-1}{2}\right\rfloor+n
\end{aligned}
$$

This contradicts (5).

Thus, $\operatorname{cr}_{D}\left(T^{i}, T^{j}\right) \neq 0$ for all $i, j=1,2, \ldots, n, \quad i \neq$ $j$. Moreover, using (2) and (3) together with $\operatorname{cr}\left(K_{6, n}\right)=$ $6\left\lfloor\frac{n}{2}\right\rfloor\left\lfloor\frac{n-1}{2}\right\rfloor$ we have

$$
\begin{aligned}
\operatorname{cr}_{D}(G(n)) & =\operatorname{cr}_{D}\left(K_{6, n}\right)+\operatorname{cr}_{D}(G)+\operatorname{cr}_{D}\left(K_{6, n}, G\right) \\
& \geq 6\left\lfloor\frac{n}{2}\right\rfloor\left\lfloor\frac{n-1}{2}\right\rfloor+\operatorname{cr}_{D}(G)+\operatorname{cr}_{D}\left(K_{6, n}, G\right) .
\end{aligned}
$$

This, together with the assumption (5), implies that

$\operatorname{cr}_{D}(G)+\operatorname{cr}_{D}\left(K_{6, n}, G\right)<n$

and hence, there is at least one subgraph $T^{i}$ which does not cross $G$ in $D$. Moreover, it implies that $G$ contains at least one internal crossing since $G$ is not outerplanar graph and furthermore, $\operatorname{cr}_{D}\left(G, K_{6, n}\right) \leq n-2$.

Without loss of generality, let $\operatorname{cr}_{D}\left(G, T^{1}\right)=0$. There exists $T^{i}, i \in\{2,3, \ldots, n\}$ such that

$\operatorname{cr}_{D}\left(G \cup T^{1}, T^{i}\right) \leq 3$. 
If otherwise, the inequality $\operatorname{cr}_{D}\left(G \cup T^{1}, T^{i}\right) \geq 4$ holds for every $i=2,3, \ldots, n$, then

$$
\begin{aligned}
\operatorname{cr}_{D}(G(n)) & =\operatorname{cr}_{D}\left(K_{6, n-1}\right)+\operatorname{cr}_{D}\left(G \cup T^{1}\right)+ \\
& +\operatorname{cr}_{D}\left(K_{6, n-1}, G \cup T^{1}\right) \\
& \geq 6\left\lfloor\frac{n-1}{2}\right\rfloor\left\lfloor\frac{n-2}{2}\right\rfloor+1+4(n-1) \\
& =6\left\lfloor\frac{n}{2}\right\rfloor\left\lfloor\frac{n-1}{2}\right\rfloor+n+\left(3 n-3-6\left\lfloor\frac{n-1}{2}\right\rfloor\right) .
\end{aligned}
$$

As $3 n-3-6\left\lfloor\frac{n-1}{2}\right\rfloor \geq 0$, in $D$ there are at least $6\left\lfloor\frac{n}{2}\right\rfloor\left\lfloor\frac{n-1}{2}\right\rfloor+$ $n$ crossings. This contradicts (5).

Assume now the subdrawing $D\left(F^{1}\right)$ of the subgraph $F^{1}=G \cup T^{1}$ induced by $D$. Since $\operatorname{cr}_{D}\left(G, T^{1}\right)=0$, all edges of $T^{1}$ are placed in one region of $D(G)$ with all six vertices of $G$ on its boundary, say outside $G$. Hence, in $D\left(F^{1}\right)$, on the boundary of every region outside $G$ there are exactly two vertices of $G$. It implies from (7) and the fact $\operatorname{cr}_{D}\left(T^{1}, T^{i}\right) \neq 0$ that, in $D$, a vertex $t_{i}, i \geq 2$, can be placed only in a region of $D\left(F^{1}\right)$ with at least four vertices of $G$ on its boundary, i.e. inside $G$. For the case $\operatorname{cr}_{D}\left(C_{5}^{e}(G)\right) \neq 0$, inside the crossed 5-cycle $C_{5}^{e}(G)$ there are regions with at most three vertices of $C_{5}^{e}(G)$. This immediately implies that there is no region with more than three vertices on its boundary in $D\left(F^{1}\right)$. The same holds if the edges of $C_{5}^{f}(G)$ cross each other. So, $\operatorname{cr}_{D}\left(C_{5}^{e}(G)\right)=0$ and $\operatorname{cr}_{D}\left(C_{5}^{f}(G)\right)=0$ and the necessary internal crossing in $G$ is forced among the edges of $C_{4}(G)$. There are only two possible drawings of $C_{4}(G)$ with one crossing yielding only two possible drawings of $G$ : the edge $a e$ is crossed by the edge $d f$ or the edge $d e$ is crossed by the edge $a f$, see Fig. 2 .
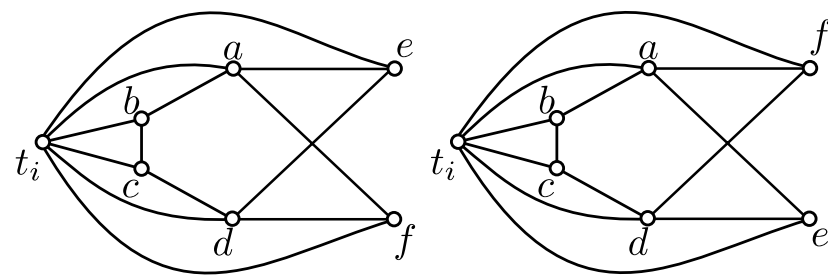

Fig. 2 Two possible drawings of $F^{1}$ containing a region with four vertices of the graph $G$.

Consider now the subdrawing of $G \cup T^{1} \cup T^{i}$ induced by $D$ and let us count the necessary crossings between the edges of $G \cup T^{1}$ and the edges of $T^{i}$. Recall that $t_{i}$ cannot be placed outside the graph $G$ and hence $t_{i}$ can be placed only in one of three possible regions. For the case of placing the vertex $t_{i}$ in the region with four vertices of $G$ on its boundary, $\operatorname{cr}_{D}\left(G \cup T^{1}, T^{i}\right)=3$ with $\operatorname{cr}_{D}\left(G, T^{i}\right)=2, \operatorname{orcr}_{D}\left(G \cup T^{1}, T^{i}\right)=4$ with $\operatorname{cr}_{D}\left(G, T^{i}\right)=2$, or $\operatorname{cr}_{D}\left(G \cup T^{1}, T^{i}\right) \geq 5$. In the other cases, $t_{i}$ is placed in a region with two vertices on its boundary (there are only two such regions). Then $T^{i}$ crosses the boundary of the region at least four times and therefore, $\operatorname{cr}\left(G, T^{i}\right) \geq 4$. Hence, $\operatorname{cr}_{D}\left(G \cup T^{1}, T^{i}\right) \geq 5$, because $\operatorname{cr}_{D}\left(T^{1}, T^{i}\right) \neq 0$. Assume that $r$ is the number of $T^{i}$ with $\operatorname{cr}_{D}\left(G \cup T^{1}, T^{i}\right)=3$ and $\operatorname{cr}_{D}\left(G, T^{i}\right)=2$ and that $s$ is the number of $T^{i}$ with
$\mathrm{cr}_{D}\left(G \cup T^{1}, T^{i}\right)=4$ and $\mathrm{cr}_{D}\left(G, T^{i}\right)=2$. The following inequality immediately follows:

$$
\begin{aligned}
\operatorname{cr}_{D}(G(n)) & =\operatorname{cr}_{D}\left(K_{6, n-1}\right)+\operatorname{cr}_{D}\left(G \cup T^{1}\right)+ \\
& +\operatorname{cr}_{D}\left(K_{6, n-1}, G \cup T^{1}\right) \\
& \geq 6\left\lfloor\frac{n-1}{2}\right\rfloor\left\lfloor\frac{n-2}{2}\right\rfloor \\
& +1+3 r+4 s+5(n-r-s-1) \\
& =6\left\lfloor\frac{n}{2}\right\rfloor\left\lfloor\frac{n-1}{2}\right\rfloor+n \\
& +\left(4 n-2 r-4-s-6\left\lfloor\frac{n-1}{2}\right\rfloor\right) .
\end{aligned}
$$

This together with $(5)$ implies

$4 n-2 r-s-4-6\left\lfloor\frac{n-1}{2}\right\rfloor<0$

and hence, $2 r+s>n+2$ for even $n$ and $2 r+s>n-1$ for odd $n$. On the other hand, $G$ contains at most $n-1$ crossings and the inequality $2 r+2 s<n-1$ must hold. This contradiction completes the proof.

\section{THE CROSSING NUMBER OF $G+P_{N}$}

The graph $G+P_{n}$ contains $G+D_{n}$ as a subgraph. Let us denote the path $t_{1} t_{2} \ldots t_{n}$ by $P_{n}$. So, it is easy to see that

$G+P_{n}=G \cup K_{6, n} \cup P_{n}=G \cup P_{n} \cup\left(\bigcup_{i=1}^{n} T^{i}\right)$.

For $n=1$, the graph $G+P_{1}$ is isomorphic to the graph $G+1$ and therefore, $\operatorname{cr}\left(G+P_{1}\right)=1$.

Theorem 3.1. $\operatorname{cr}\left(G+P_{n}\right)=6\left\lfloor\frac{n}{2}\right\rfloor\left\lfloor\frac{n-1}{2}\right\rfloor+n+1$ for $n \geq 2$.

Proof. The graph $G+P_{n}$ can be created from $G+D_{n}$ shown in Fig. 1 by adding the edges of $P_{n}$ in such way that only the edge $b c$ of $G$ is crossed by the edge $t_{\left\lfloor\frac{n}{2}\right\rfloor} t_{\left\lfloor\frac{n}{2}\right\rfloor+1}$ of $P_{n}$. This implies that $\operatorname{cr}\left(G+P_{n}\right) \leq 6\left\lfloor\frac{n}{2}\right\rfloor\left\lfloor\frac{n-1}{2}\right\rfloor+n+1$. On the other hand, $G+P_{n}$ contains $G+D_{n}$ as a subgraph and hence, by Theorem 2.1. $\operatorname{cr}\left(G+P_{n}\right) \geq 6\left\lfloor\frac{n}{2}\right\rfloor\left\lfloor\frac{n-1}{2}\right\rfloor+n$.

Suppose that there is a drawing $D$ of the graph $G+P_{n}$ with $6\left\lfloor\frac{n}{2}\right\rfloor\left\lfloor\frac{n-1}{2}\right\rfloor+n$ crossings. Then no edge of the path $P_{n}$ is crossed in $D$. In addition, as the graph $G+P_{n}$ contains $K_{6, n}$ as a subgraph with at least $6\left\lfloor\frac{n}{2}\right\rfloor\left\lfloor\frac{n-1}{2}\right\rfloor$ crossings, at most $n$ crossings appear on the edges of $G$.

Let us assume that no two edges of the subgraph $G$ cross each other. The subdrawing of $G$ induced by $D$ divides the plane into one quadrangular and two pentagonal regions and, as no edge of the path $P_{n}$ is crossed, all vertices $t_{i}, i=1,2, \ldots n$, are placed in one of the pentagonal regions. Assume now a subdrawing $D\left(F^{i}\right)$ of the subgraph $F^{i}=G \cup T^{i}$. The edges of $T^{i}$ joining the vertex $t_{i}$ with the vertices of $G$ on the boundary of the considered pentagonal region divide this region into five new regions. A vertex $t_{j}, j \neq i$, must be placed in one of them. One can easy verify that the edges of $T^{j}$ joining $t_{j}$ with the vertices of $G$ on the boundary of the pentagonal region of $D(G)$ must cross $T^{i}$ at least four times. Hence, $\operatorname{cr}_{D}\left(T^{i}, T^{j}\right) \geq 4$ for 
$i, j=1,2, \ldots, n, i \neq j$. Moreover, as $G$ is not outerplanar graph, every $T^{i}$ crosses $G$ at least once and therefore, at least $4\left(\begin{array}{c}n \\ 2\end{array}\right)+n>6\left\lfloor\frac{n}{2}\right\rfloor\left\lfloor\frac{n-1}{2}\right\rfloor+n$ crossings appear in $D$.

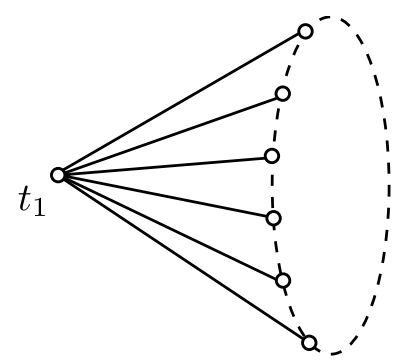

Fig. 3 The considered drawing of $G \cup T_{1}$.

This contradiction implies that the edges of $G$ cross each other and that at least one subgraph $T^{i}$, say $T^{1}$, does not cross the edges of $G$. So, all vertices of $G$ must appear on the boundary of one region in the view of the subdrawing of $G$, say outside $G$, and the subdrawing of $G \cup T^{1}$ induced by $D$ divides the plane in such a way that on the boundary of every region outside $G$ there are two vertices of $G$, see Fig. 3 in which the possible crossings among the edges of $G$ are inside the disc bounded by dotted cycle. As no edge of $P_{n}$ is crossed, all vertices $t_{i}, i=2,3, \ldots, n$, are placed outside $G$ in $D$. It is easy to verify in Fig. 3 that every $T^{i}$ crosses $G \cup T^{1}$ at least four times, but the case $\operatorname{cr}_{D}\left(G \cup T^{1}, T^{i}\right)=4$ forces that $T^{i}$ crosses $G$ at least twice. Thus, as $\operatorname{cr}_{D}(G) \neq 0$ and on the edges of $G$ there are at most $n$ crossings, at least one subgraph $T^{i}$ crosses $G \cup T^{1}$ more than four times. This implies that

$$
\begin{aligned}
\operatorname{cr}_{D}\left(G+P_{n}\right) & \geq \operatorname{cr}_{D}\left(K_{6, n-1}\right)+\operatorname{cr}_{D}\left(G+T^{1}\right) \\
& +\operatorname{cr}_{D}\left(K_{6, n-1}, G \cup T^{1}\right) \\
& \geq 6\left\lfloor\frac{n-1}{2}\right\rfloor\left\lfloor\frac{n-2}{2}\right\rfloor+1+4(n-2)+5 \\
& >6\left\lfloor\frac{n}{2}\right\rfloor\left\lfloor\frac{n-1}{2}\right\rfloor+n .
\end{aligned}
$$

This contradiction completes the proof.

\section{THE CROSSING NUMBER OF $G+C_{N}$}

The graph $G+C_{n}$ contains both $G+P_{n}$ and $G+D_{n}$ as subgraphs. Let us denote by $C_{n}$ the cycle $t_{1} t_{2} \ldots t_{n} t_{1}$ of the graph $G+C_{n}$. For $x=a, b, c, d, e, f$, let $T^{x}$ denote the subgraph of $D_{6}+C_{n}$ induced on the edges incident with the vertex $x$. One can easily see that

$$
\begin{aligned}
G+C_{n} & =G \cup K_{6, n} \cup C_{n}=G \cup\left(\bigcup_{i=1}^{n} T^{i}\right) \cup C_{n}= \\
& =G \cup\left(\bigcup_{x=a}^{f} T^{x}\right) \cup C_{n} .
\end{aligned}
$$

The proof of the main result of this section is based on the next lemma and its corollary which appeared in [14].
Lemma 4.1. ( [14]) Let $D$ be a good drawing of the graph $D_{m}+C_{n}, m \geq 2, n \geq 3$, in which no edge of $C_{n}$ is crossed and $C_{n}$ does not separate the other vertices of the graph. Then, for all $i, j=1,2, \ldots, m$, two different subgraphs $T^{i}$ and $T^{j}$ cross each other at least $\left\lfloor\frac{n}{2}\right\rfloor\left\lfloor\frac{n-1}{2}\right\rfloor$ times in D.

Corollary 4.1. ( [14]) Let $D$ be a good drawing of the graph $D_{m}+C_{n}, m \geq 2, n \geq 3$, in which the edges of $C_{n}$ cross each other and none of $r$ subgraphs $T^{i_{1}}, T^{i_{2}}, \ldots T^{i_{r}}, 2 \leq r \leq$ $m$, crosses the edges of $C_{n}$. Then, for all $j, k=1,2, \ldots, r$, two different subgraphs $T^{i_{j}}$ and $T^{i_{k}}$ cross each other at least $\left\lfloor\frac{n}{2}\right\rfloor\left\lfloor\frac{n-1}{2}\right\rfloor$ times in $D$.

Theorem 4.1. $\operatorname{cr}\left(G+C_{n}\right)=6\left\lfloor\frac{n}{2}\right\rfloor\left\lfloor\frac{n-1}{2}\right\rfloor+n+3$ for $n \geq 3$.

Proof. In Fig. 1, it is possible to add the cycle $C_{n}=$ $t_{1} t_{2} \ldots t_{n} t_{1}$ in such way that the edge $t_{\left\lfloor\frac{n}{2}\right\rfloor} t_{\left\lfloor\frac{n}{2}\right\rfloor+1}$ crosses the edge $b c$ and edge $t_{1} t_{n}$ crosses the edges $d f$ and $a e$. So, $\operatorname{cr}\left(G+C_{n}\right) \leq 6\left\lfloor\frac{n}{2}\right\rfloor\left\lfloor\frac{n-1}{2}\right\rfloor+n+3$. Assume that there is a drawing $D$ of the graph $G+C_{n}$ with at most $6\left\lfloor\frac{n}{2}\right\rfloor\left\lfloor\frac{n-1}{2}\right\rfloor+$ $n+2$ crossings. Theorem 3.1 implies that, in $D$, no edge of $C_{n}$ is crossed more than once, otherwise deleting an edge with two crossings from $C_{n}$ results in the drawing of the graph $G+P_{n}$ with fewer than $6\left\lfloor\frac{n}{2}\right\rfloor\left\lfloor\frac{n-1}{2}\right\rfloor+n+1$ crossings. Theorem 2.1 implies that there are at most two crossings on the edges of $C_{n}$. Moreover, as $G+C_{n}=$ $G \cup K_{6, n} \cup C_{n}$ and $\operatorname{cr}\left(K_{6, n}\right)=6\left\lfloor\frac{n}{2}\right\rfloor\left\lfloor\frac{n-1}{2}\right\rfloor$, in $D$ there are at most $n+2$ crossings on the edges of $G \cup C_{n}$.

Assume first that the cycle $C_{n}$ crosses $G$ in $D$. As $C_{n}$ has at most two crossings, it separates a) one vertex of degree two, or b) two vertices of degree two of $G$ from the other and, as every edge of $C_{n}$ is crossed at most once, the graph $G$ separates the vertices of $C_{n}$. Moreover, $\operatorname{cr}_{D}\left(C_{n}\right)=0$. Using (9) and the assumption $\operatorname{cr}_{D}\left(G, C_{n}\right)=2$ we have:

$$
\begin{aligned}
\operatorname{cr}_{D}\left(G+C_{n}\right) & =\operatorname{cr}_{D}(G)+\operatorname{cr}_{D}\left(K_{6, n}\right)+\operatorname{cr}_{D}\left(C_{n}\right)+ \\
& +\operatorname{cr}_{D}\left(K_{6, n}, C_{n}\right)+\operatorname{cr}_{D}\left(K_{6, n}, G\right)+\operatorname{cr}_{D}\left(G, C_{n}\right) \\
& =\operatorname{cr}_{D}(G)+\operatorname{cr}_{D}\left(K_{6, n}\right)+\operatorname{cr}_{D}\left(K_{6, n}, G\right)+2 .
\end{aligned}
$$

In the case a), $\operatorname{cr}_{D}\left(K_{6, n}\right) \geq\left(\begin{array}{l}5 \\ 2\end{array}\right)\left\lfloor\frac{n}{2}\right\rfloor\left\lfloor\frac{n-1}{2}\right\rfloor$ since, by Corollary 4.1, at least $\left(\begin{array}{l}5 \\ 2\end{array}\right)$ pairs of subgraphs $T^{x}$ and $T^{y}, x, y \in$ $\{a, b, c, d, e, f\}$, cross each other at least $\left\lfloor\frac{n}{2}\right\rfloor\left\lfloor\frac{n-1}{2}\right\rfloor$ times. Similarly, in the case b), $\operatorname{cr}_{D}\left(K_{6, n}\right) \geq\left(\begin{array}{l}4 \\ 2\end{array}\right)\left\lfloor\frac{n}{2}\right\rfloor\left\lfloor\frac{n-1}{2}\right\rfloor+$ $\left\lfloor\frac{n}{2}\right\rfloor\left\lfloor\frac{n-1}{2}\right\rfloor$ since at least $\left(\begin{array}{l}4 \\ 2\end{array}\right)$ pairs of $T^{x}$ and $T^{y}$ cross each other at least $\left\lfloor\frac{n}{2}\right\rfloor\left\lfloor\frac{n-1}{2}\right\rfloor$ times and moreover, the rest two subgraphs in the other region in the view of $C_{n}$ cross each other at least $\left\lfloor\frac{n}{2}\right\rfloor\left\lfloor\frac{n-1}{2}\right\rfloor$ times.

Suppose now that $\operatorname{cr}_{D}(G)=0$. Since $G$ is not outerplanar graph, $G$ is crossed by all subgraphs $T^{i}, i=$ $1,2, \ldots, n$, giving $\operatorname{cr}_{D}\left(K_{6, n}, G\right) \geq n$. Hence, for the case a) $\operatorname{cr}_{D}\left(G+C_{n}\right) \geq\left(\begin{array}{l}5 \\ 2\end{array}\right)\left\lfloor\frac{n}{2}\right\rfloor\left\lfloor\frac{n-1}{2}\right\rfloor+n+2$ and for the case b) $\operatorname{cr}_{D}\left(G+C_{n}\right) \geq\left(\begin{array}{l}4 \\ 2\end{array}\right)\left\lfloor\frac{n}{2}\right\rfloor\left\lfloor\frac{n-1}{2}\right\rfloor+\left\lfloor\frac{n}{2}\right\rfloor\left\lfloor\frac{n-1}{2}\right\rfloor+n+2$. Both cases contradict the assumption of the drawing $D$. This forces that $\operatorname{cr}_{D}(G) \neq 0$ and, as $\operatorname{cr}_{D}\left(G, C_{n}\right)=2$, the restriction of at most $n=2$ crossings on the edges of $G \cup C_{n}$ requires that $\operatorname{cr}_{D}\left(G, T^{i}\right)=0$ for at least one subgraph $T^{i}$. 
So, in the subdrawing $D(G)$, all vertices of $G$ are placed on the boundary of one, say outside, region. Moreover, as $G$ separates the vertices of $C_{n}$, at least one vertex of $C_{n}$ lies in the region of $D(G)$ with at most four vertices of $G$ on its boundary. This implies that there exists $T^{i}$ such that $\operatorname{cr}_{D}\left(G, T^{i}\right) \geq 2$. So, $\operatorname{cr}_{D}\left(G, K_{6, n}\right) \geq 2$. Thus, for the case a) $\operatorname{cr}_{D}\left(G+C_{n}\right) \geq 1+\left(\begin{array}{l}5 \\ 2\end{array}\right)\left\lfloor\frac{n}{2}\right\rfloor\left\lfloor\frac{n-1}{2}\right\rfloor+2+2$ and for the case b) $\operatorname{cr}_{D}\left(G+C_{n}\right) \geq 1+\left(\begin{array}{c}4 \\ 2\end{array}\right)\left\lfloor\frac{n}{2}\right\rfloor\left\lfloor\frac{n-1}{2}\right\rfloor+\left\lfloor\frac{n}{2}\right\rfloor\left\lfloor\frac{n-1}{2}\right\rfloor+2+2$. This contradiction with our assumption implies that $C_{n}$ does not cross the subgraph $G$ in $D$.

If $\operatorname{cr}_{D}\left(C_{n}\right) \neq 0$, then $n \geq 4$ and, in the view of the subdrawing of $C_{n}$, all vertices of $G$ are placed in the unique region with all vertices of $C_{n}$ on its boundary. In this case, at least five subgraphs $T^{x}, x \in\{a, b, c, d, e, f\}$, do not cross $C_{n}$ and, by Corollary 4.1, $\operatorname{cr}_{D}\left(K_{6, n}\right) \geq\left(\begin{array}{l}5 \\ 2\end{array}\right)\left\lfloor\frac{n}{2}\right\rfloor\left\lfloor\frac{n-1}{2}\right\rfloor$. For $n \geq 4$, the number of crossings in $D$ is at least $\left(\begin{array}{l}5 \\ 2\end{array}\right)\left\lfloor\frac{n}{2}\right\rfloor\left\lfloor\frac{n-1}{2}\right\rfloor+1$. This contradicts our assumption again.

So, $\operatorname{cr}_{D}\left(C_{n}\right)=0$ and the subdrawing of $C_{n}$ induced by $D$ divides the plane into two regions and all vertices of $G$ are placed in one of them. If none of the subgraphs $T^{x}, x \in\{a, b, c, d, e, f\}$, crosses $C_{n}$, then, by Lemma 4.1 in the drawing $D$ there are at least $\left(\begin{array}{l}6 \\ 2\end{array}\right)\left\lfloor\frac{n}{2}\right\rfloor\left\lfloor\frac{n-1}{2}\right\rfloor$ crossings, a contradiction with the assumption. If only one subgraph $T^{x}, x \in\{a, b, c, d, e, f\}$, crosses $C_{n}$ once or twice, then using the fact that $G$ has at least one internal crossing, the number of crossings in $D$ is at least $\left(\begin{array}{l}5 \\ 2\end{array}\right)\left\lfloor\frac{n}{2}\right\rfloor\left\lfloor\frac{n-1}{2}\right\rfloor+1+1$. This exceeds the considered number of crossings again. If two subgraphs $T^{x}$ and $T^{y}, x, y \in\{a, b, c, d, e, f\}$, cross $C_{n}$, both $T^{x}$ and $T^{y}$ cross every $T^{q}, q \in\{a, b, c, d, e, f\}, q \neq x, y$, at least $\left\lfloor\frac{n-1}{2}\right\rfloor\left\lfloor\frac{n-2}{2}\right\rfloor$ times and therefore, there are at least $\left(\begin{array}{l}4 \\ 2\end{array}\right)\left\lfloor\frac{n}{2}\right\rfloor\left\lfloor\frac{n-1}{2}\right\rfloor+8\left\lfloor\frac{n-1}{2}\right\rfloor\left\lfloor\frac{n-2}{2}\right\rfloor+2$ crossings in $D$. This is more than $6\left\lfloor\frac{n}{2}\right\rfloor\left\lfloor\frac{n-1}{2}\right\rfloor+n+2$ for $n \geq 4$.

The last possibility is that $n=3$ and two subgraphs $T^{x}$ and $T^{y}$ cross the cycle $C_{3}$ in the drawing $D$. Recall that for $\operatorname{cr}_{D}\left(K_{6,3}, C_{n}\right)=2$ and $n=3$ the inequality

$$
\begin{aligned}
\operatorname{cr}_{D}\left(G+C_{3}\right) & =\operatorname{cr}_{D}(G)+\operatorname{cr}_{D}\left(K_{6,3}\right)+\operatorname{cr}_{D}\left(K_{6,3}, G\right)+2 \\
& \geq \operatorname{cr}_{D}(G)+\operatorname{cr}_{D}\left(K_{6,3}, G\right)+6+2 \\
& =\operatorname{cr}_{D}(G)+\operatorname{cr}_{D}\left(K_{6,3}, G\right)+8
\end{aligned}
$$

holds. Thus, for the considered drawing $D$ with at most eleven crossings we have that $\operatorname{cr}_{D}(G)+\operatorname{cr}_{D}\left(K_{6,3}, G\right) \leq 3$. Assume first that $\operatorname{cr}_{D}(G)=0$. The subdrawing of $G$ induced by $D$ divides the plane into one quadrangular and two pentagonal regions and, as $\operatorname{cr}_{D}\left(G, C_{3}\right)=0$, all vertices $t_{i}$, $i=1,2,3$, are placed in one of the pentagonal regions. The simple modification of Lemma 4.1 implies that the edges of $T^{1}, T^{2}$, and $T^{3}$ joining the vertices $t_{1}, t_{2}$, and $t_{3}$ with the vertices on the boundary of this pentagonal region cross each other at least $\left(\begin{array}{l}3 \\ 2\end{array}\right)\left\lfloor\frac{5}{2}\right\rfloor\left\lfloor\frac{5-1}{2}\right\rfloor=12$ times. This contradict the assumption of the drawing.

Now, if $\operatorname{cr}_{D}(G) \neq 0$, then, by $(10), \operatorname{cr}_{D}\left(G, K_{6, n}\right) \leq 2$. This forces that at least one subgraph $T^{i}$, say $T^{1}$, does not cross the edges of $G$. Without loss of generality, assume that the vertex $t_{1}$ is placed outside $G$ in the view of the subdrawing of $G$. In this case, the subdrawing of $G \cup T^{1}$ induced by $D$ divides the plane in such a way that on the boundary of every region outside $G$ there are two vertices of $G$, see Fig. 3, in which the possible crossings among the edges of $G$ are inside the disc bounded by dot- ted cycle. All other vertices $t_{i}, i=2,3$, must be placed in the same region outside $G$. It is easy to see in Fig. 3 that the case $\operatorname{cr}_{D}\left(T^{i}, G \cup T^{1}\right)=4$ forces $\operatorname{cr}_{D}\left(T^{i}, G\right) \geq 2$ and $\operatorname{cr}_{D}\left(T^{j}, G \cup T^{1}\right) \geq 6$ for $j \neq i$. Similarly, the case $\operatorname{cr}_{D}\left(T^{i}, G \cup T^{1}\right)=5$ forces $\operatorname{cr}_{D}\left(T^{i}, G\right) \geq 1$ and $\operatorname{cr}_{D}\left(T^{j}, G \cup\right.$ $\left.T^{1}\right) \geq 5$ for $j \neq i$. Thus, $\operatorname{cr}_{D}\left(T^{1}, T^{2} \cup T^{3}\right) \geq 10$ in all possible cases, which, together with $\operatorname{cr}_{D}\left(K_{6,3}, C_{3}\right)=2$ contradicts the assumption of at most eleven crossings in the drawing of $G+C_{3}$. This completes the proof.

\section{ACKNOWLEDGEMENT}

The research was supported by the Slovak VEGA grant No. 1/0309/11 . This work was also supported by the Slovak Research and Development Agency under the contract No. APVV-0008-10.

\section{REFERENCES}

[1] BEINEKE, L. W. - RINGEISEN, R. D.: On the crossing numbers of products of cycles and graphs of order four, J. Graph Theory 4 (1980), 145-155.

[2] BHATT, S. N. - LEIGHTON, F. T.: A framework for solving VLSI graph layout problems, Journal of Computer and System Sciences 28 (1984), 300-343.

[3] BOKAL, D.: On the crossing number of Cartesian products with paths, J. Combin. Theory B 97 (2007), 381-384.

[4] DRAŽENSKÁ, E. - KLEŠČ, M.: The crossing numbers of products of cycles with 6-vertex trees, Tatra Mt. Math. Publ. 36 (2007), 109-119.

[5] GAREY, M. R. - JOHNSON, D. S.: Crossing Number is NP-Complete, SIAM Journal on Algebraic and Discrete Methods 4 (1983), 312-316.

[6] GLEBSKY, L. Y. - SALAZAR, G.: The crossing number of $C_{m} \times C_{n}$ is as conjectured for $n \geq m(m+1)$, J. Graph Theory 47 (2004), 53-72.

[7] HARARY, F. - KEINEN, P. C. - SCHWENK, A. J.: Toroidal graphs with arbitrarily high crossing numbers, Nanta Math. 6 (1973), 5867.

[8] HLINĚNÝ, P.: Crossing number is hard for cubic graphs, J. Comb. Theory B 96 (2006), 455-471.

[9] JENDROL, S. - ŠČERBOVÁ, M.: On the crossing numbers of $S_{m} \times P_{n}$ and $S_{m} \times C_{n}$, Časopis pro pěstování matematiky 107 (1982), 225-230.

[10] KLEITMAN, D. J.: The crossing number of $K_{5, n}$, Journal of Combinatorial Theory 9 (1970), 315-323.

[11] KLEŠČ, M.: The crossing numbers of products of paths and stars with 4-vertex graphs, J. Graph Theory 18 (1994), 605-614.

[12] KLEŠČ, M.: On the crossing numbers of products of stars and graphs of order five, Graphs and Comb. 17 (2001), 289-294.

[13] KLEŠČ, M.: The crossing numbers of Cartesian products of paths with 5-vertex graphs, Discrete Math. 233 (2001), 353-359. 
[14] KLEŠČ, M.: The join of graphs of crossing numbers, Electronic Notes in Discrete Mathematics 28 (2007), 349-355.

[15] KLEŠČ, M.: The crossing numbers of join of the special graph on six vertices with path and cycle, Discrete Math. 310 (2010), 1475 - 1481.

[16] KLEŠČ, M. - KOCÚROVÁ, A.: The crossing number of products of 5-vertex graphs with cycles, Discrete Math. 307 (2007), 1395-1403.

[17] KLEŠČ, M. - SCHRÖTTER, Š.: The crossing numbers of join products of paths with graphs of order four, Discuss. Math. - Graph Theory 31 (2011), 321 $-331$.

[18] KLEŠČ, M. - SCHRÖTTER, Š.: The crossing numbers of join of paths and cycles with two graphs of order five, Combinatorial Algorithms, Springer, LNCS, 7125 (2012), 160-167.

[19] SHAHROKI, F. - SÝKORA, O. - SZÉKELY, L. A. VRŤO, I.: Intersection of Curves and Crossing Number of $C_{m} \times C_{n}$ on Surfaces, Discrete \& Computational Geometry 19 (1998), 237-247.

[20] TURÁN, P.: A note of welcome, J. Graph Theory $\mathbf{1}$ (1977), 7-9.
[21] WOODALL, D. R.: Cyclic-order graphs and Zarankiewicz's crossing-number conjecture, J. Graph Theory 17 (1993), 657-671.

[22] ZARANKIEWICZ, K.: On a problem of P. Turán concerning graphs, Fund. Math 41 (1954), 137-145.

Received July 23, 2012, accepted September 24, 2012

\section{BIOGRAPHIES}

Marián Klešč is Associated Professor at the Department of Mathematics and Theoretical Informatics, Faculty of Electrical Engineering and Informatics, Technical University of Košice. He received his $\mathrm{PhD}$ degree in discrete mathematics in 1995 at P. J. Šafárik University in Košice. His scientific research is focusing on topological graph theory and crossing numbers problems.

Matúš Valo graduated in informatics from Technical University of Košice and continues the studies as a postgraduate student. His scientific research is focusing on crossing numbers problems of graphs and their algorithmic computation. 